\title{
A New Numerical Scheme for the Generalized Huxley Equation Bilge INAN
}

\author{
Department of Mathematics, Muallim Rıfat Faculty of Education, Kilis 7 Aralık University, Kilis, \\ Turkey \\ bilgeinan@kilis.edu.tr
}

Keywords: The generalized Huxley equation, Finite difference method, Exponential finite difference method, Implicit exponential finite difference method.

\begin{abstract}
In this paper, an implicit exponential finite difference method is applied to compute the numerical solutions of the nonlinear generalized Huxley equation. The numerical solutions obtained by the present method are compared with the exact solutions and obtained by other methods to show the efficiency of the method. The comparisons showed that proposed scheme is reliable, precise and convenient alternative method for solution of the generalized Huxley equation.
\end{abstract}

\section{Introduction}

Most of the problems in various field as physics, chemistry, biology, mathematics and engineering modelled by nonlinear partial differential equations. One of the nonlinear partial differential equations is the generalized Huxley equation. The generalized Huxley equation of the form;

$$
\frac{\partial u}{\partial t}-\frac{\partial^{2} u}{\partial x^{2}}=\beta u\left(1-u^{\delta}\right)\left(u^{\delta}-\gamma\right), 0 \leq x \leq 1, t \geq 0
$$

with the initial condition

$$
u(x, 0)=\left(\frac{\gamma}{2}+\frac{\gamma}{2} \tanh [\sigma \not x]\right)^{\frac{1}{\delta}}
$$

The exact solution of Eqs. (1) was derived by Wang[1] following form;

$$
u(x, t)=\left(\frac{\gamma}{2}+\frac{\gamma}{2} \tanh \left[\sigma \gamma\left(x+\left\{\frac{(1+\delta-\gamma) \rho}{2(1+\delta)}\right\} t\right)\right]\right)^{\frac{1}{\delta}}
$$

Where $\sigma=\delta \rho / 4(1+\delta), \rho=\sqrt{4 \beta(1+\delta)}$ and $\beta, \gamma$ and $\delta$ are parameters that $\beta \geq 0, \gamma \in(0,1)$. The generalized Huxley equation which describes nerve pulse propagation in nerve fibres and wall motion in liquid crystals. When $\delta=1$, Eq. (1) is reduced to the Huxley equation

$$
\frac{\partial u}{\partial t}-\frac{\partial^{2} u}{\partial x^{2}}=\beta u(1-u)(u-\gamma)
$$

Many researchers have been defined various numerical methods for numerical solutions of the generalized Huxley equation. For instance, Hashim et al.[2] used the Adomian decomposition scheme for the numerical solutions of the generalized Huxley equation. Numerical solutions of the equation was obtained using variational iteration method by Batiha et al.[7]. Hashemi et al. [6] solved the equation by means of homotopy perturbation method and Adomian decomposition method. Sari et al.[3] proposed up to tenth-order finite difference methods for solving the equation. Hemida and Mohamed [4, 5] used homotopy analysis method to obtain approximation solution of the generalized Huxley equation.

On the other hand, exponential finite difference method, the method developed in this paper, firstly was defined by Bhattacharya in 1985. Bhattacharya developed the explicit exponential finite 
difference method for solving of the heat equation[8]. After this article, Bhattacharya[9] and Handschuh and Keith[10] used exponential finite difference method for the solution of Burgers' equation. Bahadır solved the $\mathrm{KdV}$ equation by using the exponential finite difference technique[11]. Different exponential finite difference methods developed for numerical solution of the Burgers' equation by İnan and Bahadır[12-15]. Also, İnan and Bahadır[16] proposed implicit exponential finite difference method for solution of the generalized Burgers-Huxley equation.

In this paper, the generalized Huxley equation solved by implicit exponential finite difference method. Some examples are presented to show the ability of this method to solve the equation. Moreover, numerical results compared with the exact solutions and the other numerical solutions. It is clearly seen that present method offer high accuracy for the numerical solution of the nonlinear generalized Huxley equation.

\section{Implicit Exponential Finite Difference Method}

We rewritten Eq. (1) to obtain

$$
\frac{\partial u}{\partial t}=\beta u\left(1-u^{\delta}\right)\left(u^{\delta}-\gamma\right)+\frac{\partial^{2} u}{\partial x^{2}}
$$

and dividing by $u$

$$
\frac{\partial \ln u}{\partial t}=\frac{1}{u}\left(\beta u\left(1-u^{\delta}\right)\left(u^{\delta}-\gamma\right)+\frac{\partial^{2} u}{\partial x^{2}}\right)
$$

Eq. (6) rearranged and using the finite difference approximations for derivatives the equation have been taken following form

$$
U_{i}^{n+1}=U_{i}^{n} \exp \left\{\frac{k}{U_{i}^{n}}\left[\beta U_{i}^{n}\left(1-\left(U_{i}^{n}\right)^{\delta}\right)\left(\left(U_{i}^{n}\right)^{\delta}-\gamma\right)+\left(\frac{U_{i+1}^{n+1}-2 U_{i}^{n+1}+U_{i-1}^{n+1}}{h^{2}}\right)\right]\right\} .
$$

Eq. (7) is the implicit exponential finite difference method for solution of the generalized Huxley equation. Where the solution domain is discretized into cells described by the nodes $\left(x_{i}, t_{n}\right)$ in which $x_{i}=i h,(i=0,1,2, \ldots, N)$ and $t_{n}=n k,(n=0,1,2, \ldots), \quad h=\Delta x=\frac{1-0}{N}$ is the spatial mesh size and $k=\Delta t$ is the time step. Also $U_{i}^{n}$ denotes the finite difference approximation to the exact solution $u(x, t)$. Eq. (7) is system of nonlinear difference equations.

To solve the nonlinear system of equations we considered in the form

$$
\mathbf{F}(\mathbf{V})=\mathbf{0}
$$

where $\mathbf{F}=\left[f_{1}, f_{2}, \ldots, f_{N-1}\right]^{T}$ and $\mathbf{V}=\left[U_{1}^{n+1}, U_{2}^{n+1}, \ldots, U_{N-1}^{n+1}\right]^{T}$. Newton's method applied to Eq. (8) results in the following iteration:

1. Set $\mathbf{V}^{(0)}$, an initial guess.

2. For $m=0,1,2, \ldots$ until convergence do:

Solve $J\left(\mathbf{V}^{(m)}\right) \boldsymbol{\delta}^{(m)}=-F\left(\mathbf{V}^{(m)}\right) ;$

Set $\mathbf{V}^{(m+1)}=\mathbf{V}^{(m)}+\boldsymbol{\delta}^{(m)}$ where $J\left(\mathbf{V}^{(m)}\right)$ is the Jacobian matrix which is evaluated analytically. The solution at the previous time-step is taken as the initial estimate. The Newton's iteration at each time-step is stopped when $\left\|\mathbf{F}\left(\mathbf{V}^{(m)}\right)\right\|_{\infty} \leq 10^{-5}$. The convergence is generally obtained in two or three iterations. 


\section{Numerical Results}

In this section, we obtain numerical solutions of the generalized Huxley equation by implicit exponential finite difference method and exact solutions for problems. The accuracy of the proposed method is measured using the absolute error which defined by

$$
\left|u\left(x_{i}, t_{n}\right)-U\left(x_{i}, t_{n}\right)\right|
$$

All of the computational work is performed with $h=0.01$ and $k=0.0001$.

Problem 1. In Table 1,2,3 we present numerical and exact solutions for various values of $x, t$ and $\delta$ with $\beta=1, \gamma=0.001$. Also, in Table 1-3 numerical solutions obtained by implicit exponential finite difference method compared with exact solutions and those were obtained by other method[2,7]. All comparisons show that the present method offer better results than the others.

Problem 2. Absolute errors for various values of $x, t$ and $\delta$ with $\beta=0.01, \gamma=0.001$ displayed in Table 4.

Problem 3. Table 5 shows absolute errors for various values of $x, t$ and $\beta$ with $\delta=1, \gamma=0.0001$.

Problem 4. Table 6 presents absolute errors for various values of $x, t$ and $\gamma$ with $\beta=10, \delta=2$.

From all of the computed results is observed that values of the errors are very small. Also, it should be noted that the accuracy of the results decreased when $\delta$ and $\beta$ increased and the accuracy increased when $\gamma$ decreased.

Table 1. Comparison of the numerical solutions for $\delta=1$.

\begin{tabular}{|c|c|c|c|c|c|}
\hline$x$ & $t$ & {$[2]$} & {$[7]$} & Present Method & Exact \\
\hline 0.1 & 0.05 & $5.00005184 \times 10^{-4}$ & $5.00005184 \times 10^{-4}$ & $5.00012528 \times 10^{-4}$ & $5.00030171 \times 10^{-4}$ \\
\hline & 0.1 & $4.99992690 \times 10^{-4}$ & $4.99992690 \times 10^{-4}$ & $5.00010148 \times 10^{-4}$ & $5.00042665 \times 10^{-4}$ \\
\hline & 1 & $4.99767803 \times 10^{-4}$ & $4.99767803 \times 10^{-4}$ & $5.00006434 \times 10^{-4}$ & $5.00267553 \times 10^{-4}$ \\
\hline & 5 & - & - & $5.00006433 \times 10^{-4}$ & $5.01267050 \times 10^{-4}$ \\
\hline & 10 & - & - & $5.00006433 \times 10^{-4}$ & $5.02516406 \times 10^{-4}$ \\
\hline & & & & & \\
\hline 0.5 & 0.05 & $5.00075895 \times 10^{-4}$ & $5.00075895 \times 10^{-4}$ & $5.00076825 \times 10^{-4}$ & $5.00100882 \times 10^{-4}$ \\
\hline & 0.1 & $5.00063401 \times 10^{-4}$ & $5.00063401 \times 10^{-4}$ & $5.00069175 \times 10^{-4}$ & $5.00113376 \times 10^{-4}$ \\
\hline & 1 & $4.99838513 \times 10^{-4}$ & $4.99838513 \times 10^{-4}$ & $5.00057156 \times 10^{-4}$ & $5.00338263 \times 10^{-4}$ \\
\hline & 5 & - & - & $5.00057154 \times 10^{-4}$ & $5.01337760 \times 10^{-4}$ \\
\hline & 10 & - & - & $5.00057154 \times 10^{-4}$ & $5.02587115 \times 10^{-4}$ \\
\hline & & & & & \\
\hline 0.9 & 0.05 & $5.00146605 \times 10^{-4}$ & $5.00146605 \times 10^{-4}$ & $5.00153949 \times 10^{-4}$ & $5.00171593 \times 10^{-4}$ \\
\hline & 0.1 & $5.00134111 \times 10^{-4}$ & $5.00134111 \times 10^{-4}$ & $5.00151570 \times 10^{-4}$ & $5.00184087 \times 10^{-4}$ \\
\hline & 1 & $4.99909224 \times 10^{-4}$ & $4.99909224 \times 10^{-4}$ & $5.00147855 \times 10^{-4}$ & $5.00408974 \times 10^{-4}$ \\
\hline & 5 & - & - & $5.00147855 \times 10^{-4}$ & $5.01408470 \times 10^{-4}$ \\
\hline & 10 & - & - & $5.00147855 \times 10^{-4}$ & $5.02657824 \times 10^{-4}$ \\
\hline
\end{tabular}


Table 2. Comparison of the numerical solutions for $\delta=2$.

\begin{tabular}{|c|c|c|c|c|c|}
\hline$x$ & $t$ & {$[2]$} & {$[7]$} & Present Method & Exact \\
\hline 0.1 & 0.05 & $2.23607664 \times 10^{-2}$ & $2.23607664 \times 10^{-2}$ & $2.23610949 \times 10^{-2}$ & $2.23618841 \times 10^{-2}$ \\
\hline & 0.1 & $2.23602076 \times 10^{-2}$ & $2.23602076 \times 10^{-2}$ & $2.23609885 \times 10^{-2}$ & $2.23624429 \times 10^{-2}$ \\
\hline & 1 & $2.23501462 \times 10^{-2}$ & $2.23501490 \times 10^{-2}$ & $2.23608224 \times 10^{-2}$ & $2.23724988 \times 10^{-2}$ \\
\hline & & & & & \\
\hline 0.5 & 0.05 & $2.23633483 \times 10^{-2}$ & $2.23633483 \times 10^{-2}$ & $2.23633899 \times 10^{-2}$ & $2.23644658 \times 10^{-2}$ \\
\hline & 0.1 & $2.23627895 \times 10^{-2}$ & $2.23627895 \times 10^{-2}$ & $2.23630477 \times 10^{-2}$ & $2.23650245 \times 10^{-2}$ \\
\hline & 1 & $2.23527292 \times 10^{-2}$ & $2.23527320 \times 10^{-2}$ & $2.23625102 \times 10^{-2}$ & $2.23750792 \times 10^{-2}$ \\
\hline & & & & & \\
\hline 0.9 & 0.05 & $2.23659298 \times 10^{-2}$ & $2.23659298 \times 10^{-2}$ & $2.23611415 \times 10^{-2}$ & $2.23670472 \times 10^{-2}$ \\
\hline & 0.1 & $2.23653711 \times 10^{-2}$ & $2.23653711 \times 10^{-2}$ & $2.23661518 \times 10^{-2}$ & $2.23676058 \times 10^{-2}$ \\
\hline & 1 & $2.23553120 \times 10^{-2}$ & $2.23553148 \times 10^{-2}$ & $2.23659856 \times 10^{-2}$ & $2.23776594 \times 10^{-2}$ \\
\hline
\end{tabular}

Table 3. Comparison of the numerical solutions for $\delta=3$.

\begin{tabular}{|c|c|c|c|c|c|}
\hline$x$ & $t$ & {$[2]$} & {$[7]$} & Present Method & Exact \\
\hline 0.1 & 0.05 & $7.93700531 \times 10^{-2}$ & $7.93700531 \times 10^{-2}$ & $7.93712192 \times 10^{-2}$ & $7.93740204 \times 10^{-2}$ \\
\hline & 0.1 & $7.93680693 \times 10^{-2}$ & $7.93680695 \times 10^{-2}$ & $7.93708414 \times 10^{-2}$ & $7.93760039 \times 10^{-2}$ \\
\hline & 1 & $7.93323439 \times 10^{-2}$ & $7.93323637 \times 10^{-2}$ & $7.93702517 \times 10^{-2}$ & $7.94116901 \times 10^{-2}$ \\
\hline & & & & & \\
\hline 0.5 & 0.05 & $7.93779893 \times 10^{-2}$ & $7.93779894 \times 10^{-2}$ & $7.93781370 \times 10^{-2}$ & $7.93819558 \times 10^{-2}$ \\
\hline & 0.1 & $7.93760059 \times 10^{-2}$ & $7.93760061 \times 10^{-2}$ & $7.93769226 \times 10^{-2}$ & $7.93839389 \times 10^{-2}$ \\
\hline & 1 & $7.93402876 \times 10^{-2}$ & $7.93403074 \times 10^{-2}$ & $7.93750142 \times 10^{-2}$ & $7.94196179 \times 10^{-2}$ \\
\hline & & & & & \\
\hline 0.9 & 0.05 & $7.93859239 \times 10^{-2}$ & $7.93859240 \times 10^{-2}$ & $7.93870895 \times 10^{-2}$ & $7.93898896 \times 10^{-2}$ \\
\hline & 0.1 & $7.93839409 \times 10^{-2}$ & $7.93839411 \times 10^{-2}$ & $7.93867117 \times 10^{-2}$ & $7.93918724 \times 10^{-2}$ \\
\hline & 1 & $7.93482298 \times 10^{-2}$ & $7.93482496 \times 10^{-2}$ & $7.93861219 \times 10^{-2}$ & $7.94275442 \times 10^{-2}$ \\
\hline
\end{tabular}

Table 4. Absolute errors for various values of $x, t$ and $\delta$.

\begin{tabular}{|c|c|c|c|c|c|}
\hline$x$ & $t$ & $\delta=1$ & $\delta=2$ & $\delta=4$ & $\delta=8$ \\
\hline 0.1 & 0.2 & $5.98609958 \times 10^{-12}$ & $8.46576246 \times 10^{-9}$ & $1.00676725 \times 10^{-8}$ & $3.47185291 \times 10^{-8}$ \\
\hline & 0.5 & $1.36171298 \times 10^{-11}$ & $1.92578438 \times 10^{-9}$ & $2.29018539 \times 10^{-8}$ & $3.47185291 \times 10^{-8}$ \\
\hline & 0.8 & $2.11235696 \times 10^{-11}$ & $2.98737232 \times 10^{-9}$ & $3.55264885 \times 10^{-8}$ & $1.22513605 \times 10^{-7}$ \\
\hline & & & & & \\
\hline 0.5 & 0.2 & $7.67609424 \times 10^{-12}$ & $1.08558054 \times 10^{-9}$ & $1.29099487 \times 10^{-8}$ & $4.45200874 \times 10^{-8}$ \\
\hline & 0.5 & $1.56009554 \times 10^{-11}$ & $2.20634211 \times 10^{-9}$ & $2.62382731 \times 10^{-8}$ & $9.04829223 \times 10^{-8}$ \\
\hline & 0.8 & $2.31226336 \times 10^{-11}$ & $3.27008390 \times 10^{-9}$ & $3.88885023 \times 10^{-8}$ & $1.34107312 \times 10^{-7}$ \\
\hline & & & & & \\
\hline 0.9 & 0.2 & $5.98609958 \times 10^{-12}$ & $8.46574454 \times 10^{-9}$ & $1.00676241 \times 10^{-8}$ & $3.47182392 \times 10^{-8}$ \\
\hline & 0.5 & $1.36171298 \times 10^{-11}$ & $1.92578014 \times 10^{-9}$ & $2.29017378 \times 10^{-8}$ & $7.89766967 \times 10^{-8}$ \\
\hline & 0.8 & $2.11235696 \times 10^{-11}$ & $2.98736563 \times 10^{-9}$ & $3.55263047 \times 10^{-8}$ & $1.22512503 \times 10^{-7}$ \\
\hline
\end{tabular}


Table 5. Absolute errors for various values of $x, t$ and $\beta$.

\begin{tabular}{|c|c|c|c|c|}
\hline$x$ & $t$ & $\beta=1$ & $\beta=10$ & $\beta=100$ \\
\hline 0.1 & 0.2 & $5.98610074 \times 10^{-10}$ & $5.98610073 \times 10^{-9}$ & $5.98609884 \times 10^{-8}$ \\
\hline & 0.5 & $1.36171311 \times 10^{-9}$ & $1.36171308 \times 10^{-8}$ & $1.36171038 \times 10^{-7}$ \\
\hline & 0.8 & $2.11235710 \times 10^{-9}$ & $2.11235698 \times 10^{-8}$ & $2.11234614 \times 10^{-7}$ \\
\hline & & & & \\
\hline 0.5 & 0.2 & $7.67609736 \times 10^{-10}$ & $7.67609733 \times 10^{-9}$ & $7.67609449 \times 10^{-8}$ \\
\hline & 0.5 & $1.56009590 \times 10^{-9}$ & $1.56009586 \times 10^{-8}$ & $1.56009268 \times 10^{-7}$ \\
\hline & 0.8 & $2.31226374 \times 10^{-9}$ & $2.31226359 \times 10^{-8}$ & $2.31225157 \times 10^{-7}$ \\
\hline & & & & \\
\hline 0.9 & 0.2 & $5.98610073 \times 10^{-10}$ & $5.98610063 \times 10^{-9}$ & $5.98609694 \times 10^{-8}$ \\
\hline & 0.5 & $1.36171310 \times 10^{-9}$ & $1.36171304 \times 10^{-8}$ & $1.36170938 \times 10^{-7}$ \\
\hline & 0.8 & $2.11235709 \times 10^{-9}$ & $2.11235689 \times 10^{-8}$ & $2.11234368 \times 10^{-8}$ \\
\hline
\end{tabular}

Table 6. Absolute errors for various values of $x, t$ and $\gamma$.

\begin{tabular}{|c|c|c|c|c|}
\hline$x$ & $t$ & $\gamma=10^{-3}$ & $\gamma=10^{-4}$ & $\gamma=10^{-5}$ \\
\hline 0.1 & 0.2 & $2.67497835 \times 10^{-5}$ & $8.46534623 \times 10^{-7}$ & $2.67717730 \times 10^{-8}$ \\
\hline & 0.5 & $6.08056711 \times 10^{-5}$ & $1.92554969 \times 10^{-6}$ & $6.08997590 \times 10^{-8}$ \\
\hline & 0.8 & $9.42531105 \times 10^{-5}$ & $2.98678386 \times 10^{-6}$ & $9.44700253 \times 10^{-8}$ \\
\hline & & & & \\
\hline 0.5 & 0.2 & $3.42961873 \times 10^{-5}$ & $1.08551074 \times 10^{-6}$ & $3.43299273 \times 10^{-8}$ \\
\hline & 0.5 & $6.96546632 \times 10^{-5}$ & $2.20604578 \times 10^{-6}$ & $6.97719183 \times 10^{-8}$ \\
\hline & 0.8 & $1.03157943 \times 10^{-4}$ & $3.26939677 \times 10^{-6}$ & $1.03410216 \times 10^{-7}$ \\
\hline & & & & \\
\hline 0.9 & 0.2 & $2.67321561 \times 10^{-5}$ & $8.46479088 \times 10^{-7}$ & $2.67715975 \times 10^{-8}$ \\
\hline & 0.5 & $6.07632881 \times 10^{-5}$ & $1.92541661 \times 10^{-6}$ & $6.08993385 \times 10^{-8}$ \\
\hline & 0.8 & $9.41857900 \times 10^{-5}$ & $2.98657318 \times 10^{-6}$ & $9.44693598 \times 10^{-8}$ \\
\hline
\end{tabular}

From comparisons of the numerical results with the exact solutions it is deduced that the proposed method gives highly accurate solutions. The accuracy of the method is measured in terms of the error norm defined by

$$
E=\left[\frac{\sum_{i=0}^{N}\left|u_{i}-U_{i}\right|^{2}}{\sum_{i=0}^{N}\left|u_{i}\right|^{2}}\right]^{\frac{1}{2}} .
$$

The rates of convergence of the method, computed using

$$
\text { rate }=\frac{\log \left(E^{h} / E^{h / 2}\right)}{\log (2)}
$$

where $E^{h}$ and $E^{h / 2}$ are the errors defined in Eq. (9) with the grid size $h$ and $h / 2$, respectively. Rate of convergence at $\delta=1$ and $t=1$ for the Problem 1 is shown in Table 7. From the table, we observe that the proposed method is second order accurate in space. From this table, it can be seen that errors approach to zero as the mesh refines, which shows that the scheme is consistent. 
Table 7. Rate of convergence for $\delta=1$ at $t=1$.

\begin{tabular}{|c|c|}
\hline$N$ & Rate \\
\hline 2 & 0.02802305 \\
\hline 4 & 0.01363943 \\
\hline 8 & 0.00675767 \\
\hline 16 & 0.00336688 \\
\hline 32 & 0.00168088 \\
\hline 64 & 0.00083985 \\
\hline
\end{tabular}

\section{Conclusion}

In this paper, implicit exponential finite difference method has been successfully used to study the generalized Huxley equation. Numerical solutions for different test problems are given using tables. According to the results presented in these tables, the present method offers high accuracy for the numerical solution of the nonlinear generalized Huxley equation. In the other hand, as can be seen from Table 1-3, results obtained by the implicit exponential finite difference scheme has better than results obtained from the other numerical schemes. The proposed method are seen to be very good alternative to existing methods for such problems.

\section{Acknowledgement}

This paper is presented at the conference of ICNASE' 16.

\section{References}

[1] X. Y. Wang, Z. S. Zhu, Y. K. Lu, Solitary wave solutions of the generalized Burgers-Huxley equation, J. Phys. A: Math. Gen. 23 (1990) 271-274.

[2] I. Hashim, M. S. M. Noorani, B. Batiha, A note on the Adomian decomposition method for the generalized Huxley Equation, Appl. Math. Comput. 181 (2006) 1439-1445.

[3] M. Sari, G. Gürarslan, A. Zeytinoglu, High-order finite difference schemes for numerical solutions of the generalized Burgers-Huxley equation, Numerical Methods for Partial Differential Equations. 27 (2010) 1313-1326.

[4] K. Hemida, M.S. Mohamed, Numerical simulation of the generalized Huxley equation by homotopy analysis method, Journal of Applied Functional Analysis. 5(2010) 344-350.

[5] K. Hemida, M.S. Mohamed, Application of homotopy analysis method to fractional order generalized Huxley equation, Journal of Applied Analysis. 7 (2012) 367-372.

[6] S. H. Hashemi, H. R. M. Daniali, D. D. Ganji, Numerical simulation of the generalized Huxley equation by He's homotopy perturbation method, Appl. Math. Comput. 192 (2007) 157-161.

[7] B. Batiha, M. S. M. Noorani, I. Hashim, Numerical simulation of the generalized Huxley equation by He's variational iteration method, Appl. Math. Comput. 186 (2007) 1322-1325.

[8] M. C. Bhattacharya, An explicit conditionally stable finite difference equation for heat conduction problems, Int. J. Num. Meth. Eng. 21 (1985) 239-265.

[9] M. C. Bhattacharya, Finite difference solutions of partial differential equations, Commun. Appl. Numer. Meth. 6 (1990) 173-184.

[10] R. F. Handschuh, T. G. Keith, Applications of an exponential finite-difference technique, Numer. Heat Transfer. 22 (1992) 363-378.

[11] A. R. Bahadır, Exponential finite-difference method applied to Korteweg-de Vries equation for small times, Appl. Math. Comput. 160 (2005) 675-682.

[12] B. İnan, A. R. Bahadır, Numerical solution of the one-dimensional Burgers equation: Implicit and fully implicit exponential finite difference methods, Pramana J. Phys. 81 (2013) 547-556. 
[13] B. İnan, A. R. Bahadır, An explicit exponential finite difference method for the Burgers' equation, European International Journal of Science and Technology. 2 (2013) 61-72.

[14] B. İnan, A. R. Bahadır, A numerical solution of the Burgers' equation using a Crank-Nicolson exponential finite difference method, Journal of Mathematical and Computational Science. 4 (2014) 849-860.

[15] B. İnan, A. R. Bahadır, Two Different Exponential Finite Difference Methods for Numerical Solutions of the Linearized Burgers' Equation, International Journal of Modern Mathematical Sciences. 13 (2015) 449-461.

[16] B. İnan, A. R. Bahadır, Numerical solutions of the generalized Burgers-Huxley equation by implicit exponential finite difference method, Journal of Applied Mathematics, Statistic and Informatics. 11 (2015) 57-67. 